\title{
Prevention of Post-Resection Acute Liver Failure by Various Methods of Cyanocobalamin Administration in the Experiment
}

\author{
Anastasiya Yu. Laptiyova ${ }^{1}$; Alexandr A. Andreev, PhD, ScD ${ }^{1}$; \\ Alexandr A. Glukhov, $\mathrm{PhD}, \mathrm{ScD}^{1}$; Dmitry A. Atiakshin, $\mathrm{PhD}^{2}$; Anton P. Ostroushko, $\mathrm{PhD}^{1^{*}}$; \\ Sergey N. Boyev, $\mathrm{PhD}^{1}$; Tatyana G. Nikishina, $\mathrm{PhD}^{1}$; Elena V. Mikulich, $\mathrm{PhD}^{1}$; \\ Pyotr I. Koshelev, $\mathrm{PhD}, \mathrm{ScD}^{1}$; Ekaterina A. Andreeva ${ }^{1}$ \\ ${ }^{I}$ N.N. Burdenko Voronezh State Medical University \\ ${ }^{2}$ Research Institute of Experimental Biology and Medicine, N. N. Burdenko Voronezh State Medical University \\ Voronezh, the Russian Federation
}

\begin{abstract}
Background: Surgical treatment of massive hepatic formations is an acute problem of operative hepatology, often being the only way to increase the life expectancy of patients. However, even nowadays, liver surgeries are considered to be traumatic, high-risk interventions due to the development of a potent body stress response induced by tissue damage, risk of severe intra- and postoperative complications, and high mortality. High mortality rates, especially after extensive liver resections, are more often associated with bleeding, bile flow from a liver section with the subsequent development of peritonitis, intoxication, and posthepatectomy liver failure. The aim of this study was to perform intraoperative, post-resection prevention of acute liver failure (ALF) by applying cyanocobalamin in the experiment.

Methods and Results: The study included 96 sexually mature male Wistar rats, which were divided into 4 groups, 24 animals in each group. A conventional $70 \%$ hepatectomy was performed on animals of all groups. Prevention of post-hepatectomy liver failure (PHLF) was not performed in animals of the control group (CG) 1 ; animals of CG2 received $1 \mathrm{ml}$ of $0.9 \%$ sodium chloride solution that was injected into the preserved hepatic lobes intraoperatively; animals of the experimental group (EG) 1 received 10 intrahepatic injections of $0.1 \mathrm{ml}$ of cyanocobalamin $(200 \mu \mathrm{g} / \mathrm{ml})$; animals of EG2 received $1 \mathrm{ml}$ of cyanocobalamin $(200 \mu \mathrm{g} / \mathrm{ml})$ intraperitoneally. The general condition of the animals (activity, appetite), healing time of the postoperative wound, and weight of the regenerated liver were assessed after the experiment. Biochemical methods included assessment of the indices of the cytolysis syndrome, cholestatic syndrome, the syndrome of hepatic cell failure, as well as the analysis of oxidative stress parameter and sevaluation of the expression of transforming growth factor beta (TGF- $\beta$ ).

On Day 5 after hepatic resection, a significant improvement in the general condition of the animals (increased appetite and activity) was noted in EG1 and EG2. Intraoperative, intrahepatic administration of cyanocobalamin can increase the TGF- $\beta$ expression by 2.5-3 times, affecting the proliferative activity of hepatocytes and providing recovery to $96.15 \pm 4.31 \%$ of the initial liver weight by Day 14 after hepatectomy

Conclusion: Intraoperative, intrahepatic administration of cyanocobalamin prevents the development of ALF in the early postoperative period and ensures restoration of the anatomical and functional integrity of the liver. (International Journal of Biomedicine. 2020;10(3):257-261.)
\end{abstract}

Key Words: acute liver failure $\bullet$ intraoperative prevention $\bullet$ cyanocobalamin $\bullet$ hepatectomy

\section{Abbreviations}

ALF, acute liver failure; AST, aspartate aminotransferase; ALT, alanine aminotransferase; ALP, alkaline phosphatase; BUN, blood urea nitrogen; CB, conjugated bilirubin; Cr, creatinine; GGTP, gamma-glutamyl transpeptidase; GL, glucose; GPx, glutathione peroxidase; HDL, high density lipoprotein; LDL, low density lipoprotein; MDA, malondialdegyde; PHLF, posthepatectomy liver failure; SOD, superoxide dismutase; TBIL, total bilirubin; TC, total cholesterol; TP, total protein; Tg, thyroglobulin; TGF- $\beta$, transforming growth factor beta; VLDL, very LDL. 


\section{Introduction}

Surgical treatment of massive hepatic formations is an acute problem of operative hepatology, often being the only way to increase the life expectancy of patients. ${ }^{(1)}$ Laparoscopic technologies in hepatology are advancing at a slower rate than surgical technologies of other abdominal organs, since the large size and localization features of the liver do not always allow for a full laparoscopic examination of its surface and impede free instrumental access, especially to the posterior segments. Thus, an open liver resection remains the main surgical option. ${ }^{(2)}$ An anatomical liver resection still appears to be the common method of radical treatment for patients with focal liver formations.(3) However, even nowadays, liver surgeries are considered to be traumatic, high-risk interventions due to the development of a potent body stress response induced by tissue damage, risk of severe intra- and postoperative complications $(6 \%-36 \%)$, and high mortality even in patients who do not have structural and functional liver disorders. ${ }^{(3,4)}$ High mortality rates, especially after extensive liver resections, are more often associated with bleeding, bile flow from a liver section with the subsequent development of peritonitis, intoxication, and PHLF. ${ }^{(5)}$ To date, PHLF develops in $32 \%-60 \%$ of cases. ${ }^{(6,7)} \mathrm{A}$ significant increase in the risk of developing fatal PHLF is manifested in patients with chronic organ diseases who are subjected to a resection of $60 \%$ or more of the functioning parenchyma, as well as in patients with intact parenchyma who are subjected to a resection of $75 \%-80 \%$ of the organ. ${ }^{(6)}$

The risk of developing ALF is due to several factors. First of all, ALF is a functional insufficiency of the remaining section of the liver parenchyma often resulting from initial dystrophic or cirrhotic changes. Hypoxia, which can be circulatory (due to ischemia caused by compression of the hepatoduodenal ligament - the Pringle maneuver), hemic (due to massive blood loss), or mixed, appears to be the risk factor of greatest importance.

For the prevention and treatment of ALF, various drugs with hepatoprotective properties are widely used in the scheme of pre- and postoperative infusion therapy, many of them including cyanocobalamin. ${ }^{(8)}$ However, currently, there is no single approach to reducing the risk of ALF development in the early postoperative period by activating hepatocyte proliferation.

The aim of this study was to perform intraoperative, post-resection prevention of ALF by applying cyanocobalamin in the experiment.

\section{Materials and Methods}

The study included 96 sexually mature male Wistar rats, which were divided into 4 groups, 24 animals in each group. A conventional $70 \%$ hepatectomy was performed on animals of all groups, according to the model suggested by G. Higgins. ${ }^{(9)}$ The operation was performed under intramuscular anesthesia using the anesthetic Zoetil-100 at a dose of $8 \mathrm{mc} / \mathrm{kg}$ in aseptic conditions. An oblique hypochondrium incision from the top of the xiphoid process to the free edge of the $11^{\text {th }}$ rib was used as a surgical access. The removed lobes were mobilized by crossing the venous ligament. The previously isolated vascular pedicle was ligated with suturing ligature. The left and medial lobes of the liver were cut off above the level of the sutured ligature. Prevention of PHLF was not performed in animals of the control group (CG) 1 ; animals of CG2 received $1 \mathrm{ml}$ of $0.9 \%$ sodium chloride solution that was injected into the preserved hepatic lobes intraoperatively; animals of the experimental group (EG) 1 received 10 intrahepatic injections of $0.1 \mathrm{ml}$ of cyanocobalamin $(200 \mu \mathrm{g} / \mathrm{ml})$ (certificate of acceptance No. 2019113614 of 05/06/2019); animals of EG2 received $1 \mathrm{ml}$ of cyanocobalamin $(200 \mu \mathrm{g} / \mathrm{ml})$ intraperitoneally. The wound was sutured in layers: the muscles were sutured with simple locking stiches; the skin was sutured with simple interrupted stitches. Polyhydroxy Acetyl suturing material was applied at all stages of the experiment. The animals were removed from the experiment on Days 1, 5, 7 and 14 after the intervention.

The general condition of the animals (activity, appetite), healing time of the postoperative wound, and weight of the regenerated liver were assessed after the experiment. The weight of the regenerated liver was calculated using the formula: $\mathrm{M}_{\text {initial }}=\mathrm{M}_{\text {resulted }} \times 3 / 2$. Biochemical methods included assessment of the blood levels of AST, ALT, TBIL, CB, ALP, GGTP, TC, TP, HDL, LDL, VLDL, Tg, GL, Cr, BUN, GPx, SOD, MDA, and TGF- $\beta$.

Work on the animals was done in compliance with the principles of the Helsinki Declaration on the humane treatment of animals, stated in normative documents of the European community(86/609/EU), Manual on Experimental (Preclinical) Study of New Pharmacological Substances, and "Good laboratory practice" (MHRF Order No. $708 \mathrm{H}$ of 23.08.2010).

Statistical analysis was performed using the Statistica 6.1 software package (Stat-Soft Inc., USA). The normality of distribution of continuous variables was tested by the Kolmogorov-Smirnov test with the Lilliefors correction and Shapiro-Wilk test. For descriptive analysis, results are presented as mean \pm standard deviation (SD) and median (Me). For data with normal distribution, inter-group comparisons were performed using Student's t-test. Mann-Whitney U test and Wilcoxon criterion were used to compare means of variables not normally distributed. A probability value of $P<0.05$ was considered statistically significant.

\section{Results and Discussion}

The general condition of the animals in all experimental groups on Day 1 after the operation was specified as a state of moderate severity; there was a lack of appetite and a pronounced decrease in activity. Compared to the initial parameters, the liver weight was $32.44 \pm 3.01 \%$ in CG1, $35.16 \pm 2.86 \%$ in $\mathrm{CG} 2,35.01 \pm 3.12 \%$ in $\mathrm{EG} 1$, and $38.41 \pm 4.05 \%$ in $\mathrm{EG} 2$. On Day 5 after hepatic resection, a significant improvement in the general condition of the animals (increased appetite and activity) was noted in EG1 and EG2; the animals of CG1 and CG2 remained inactive and their appetite did not increase significantly. Compared to the initial parameters, the liver weight was $41.87 \pm 2.43 \%$ in CG1, $44.11 \pm 3.80 \%$ in 
$\mathrm{CG} 2,59.45 \pm 3.48 \%$ in $\mathrm{EG} 1$, and $52.05 \pm 2.13 \%$ in $\mathrm{EG} 2$. On Day 7 of the study, the general condition of the animals of all experimental groups was specified as close to satisfactory; their appetite and activity were not reduced. Complete healing of the postoperative wound was observed; the sutures were removed. Compared to the initial parameters, the liver weight was $64.15 \pm 3.07 \%$ in CG1, $70.18 \pm 2.46 \%$ in CG2, $88.56 \pm 4.02 \%$ in EG1, and $77.34 \pm 3.43 \%$ in EG2. On Day 14 of the study, the general condition of the animals that underwent the operation in all experimental groups did not differ from the state of intact animals. Almost complete restoration of the animals' hair was noted at the site of the postoperative scar. Compared to the initial parameters, the liver weight was $82.04 \pm 3.48 \%$ in $\mathrm{CG} 1,80.54 \pm 4.01 \%$ in $\mathrm{CG} 2,96.15 \pm 4.31 \%$ in $\mathrm{EG} 1$, and $87.09 \pm 3.91 \%$ in EG2.

When assessing indicators of the blood biochemical tests, the cytolysis syndrome was the most pronounced from Day 1 to Day 7 of the study. By Day 14, there were no symptoms of cytolysis in animals of all experimental groups (Fig.1).
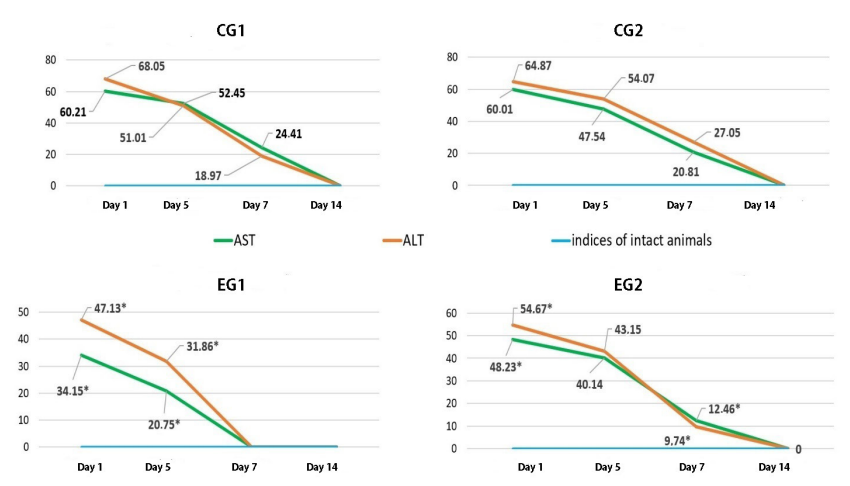

Fig. 1. Dynamics of indicators of the cytolysis syndrome, \%

On Day 1 after hepatic resection, in all groups of animals, indices of cholestatic syndrome significantly exceeded the indices of intact animals. On Day 5 of the experiment, indicators of cholestatic syndrome decreased insignificantly. It should be noted that the increase in TBIL in animals of all groups occurred mainly due to $\mathrm{CB}$, which is associated with a decrease in the functional capabilities of the hepatocytes of the resected liver. The absence of a significant increase in un-CB in the blood of animals excludes the presence of necrosis of liver cells and entry of bilirubin glucuronide into the bloodstream. On Day 7 after the operation in animals of EG1, the levels of ALP and GGTPs were normalized. On Day 14 after the operation, the indicators of cholestatic syndrome slightly exceeded normal parameters in animals of CG1 (Fig.2). The TC level in animals of CG1, CG2 and EG2 was lower than parameters of intact animals throughout the experiment. It should be noted that in animals of EG1, the TC level returned to normal value on Day 7 after the operation (Fig.2).

When assessing the syndrome of hepatic cell failure, there was a significant decrease in parameters characterizing the synthetic (TP, lipid profile), metabolic (GL) and detoxification (Cr, BUN) functions of the liver in all groups of animals on Day 1 after hepatic resection. On Day 5 , the proteinsynthetic function was reduced. The TC level was reduced in all groups of animals, while there was also a decrease in the levels of HDL, LDL, VLDL and Tg, which evidenced damage to the synthetic and energy liver functions. It should be noted that the decrease in TC might also be associated with the presence of $\beta$-muricholic acid in rats; this contributes to the rapid excretion of the excess cholesterol from the body. An increase in the level of blood GL is manifested due to a decrease in glucokinase activity in the first days after surgery, and as a result, damage to carbohydrate metabolism. On Day 5 of the study, a decrease in detoxification liver function was observed in all groups of animals. Compared to parameters of intact animals, on Day 7 after hepatic resection, parameters of lipid profile, GL, Cr, and BUN parameters were normalized in EG1 animals. On Day 14 of the study, parameters of $\mathrm{Cr}$ and BUN were normalized in animals of CG1, CG2 and EG2. In animals of EG1, only one parameter differed from that of intact animals - TP level decreased by $7.05 \pm 1.18 \%$ (Table 1 ).

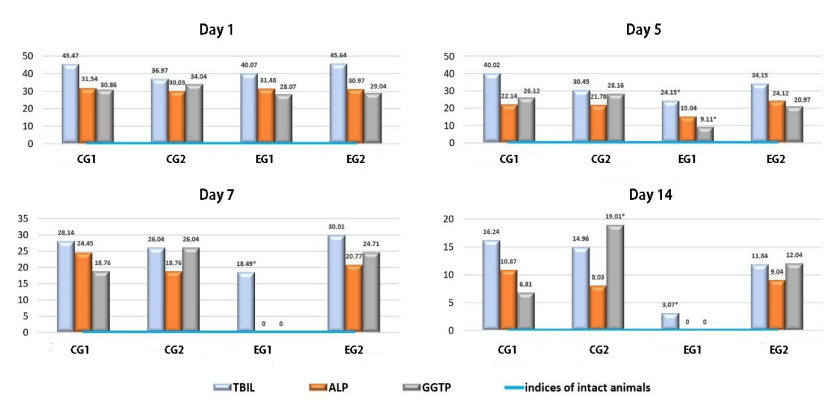

Fig. 2. Dynamics of indicators of the cholestatic syndrome, $\%$

The biochemical study demonstrated that the highest severity of the cytolytic syndrome was from Day 1 to Day 5 after hepatic resection, a fact associated not only with intraoperative damage to the liver tissue, but also with the activation of apoptosis of damaged cells in the first days of the postoperative period. With intrahepatic and intraperitoneal administration of cyanocobalamin, cytolysis indices were normalized on Day 7 of the experiment, compared to the control groups. This normalization was associated with the restoration of the morphological structure of hepatocytes, membrane integrity and total liver weight. Cholestatic syndrome was more pronounced on Day 1 after hepatic resection. In animals of EG1, the levels of ALP, GGTP, and TC were normalized on Day 7 of the experiment; in animals of the other groups these parameters differed from the norm. A similar picture was observed on Day 14 after the operation. The results obtained were associated with severe functional liver failure in the postoperative period since in animals of EG1 the largest restoration of the initial liver weight was observed $(88.56 \pm 4.02 \%)$ on Day 7 of the study, and abnormality of blood biochemical parameters was compensated. Hepatic cell failure syndrome was the most pronounced from Day 1 to Day 5 after the operation: the 
synthetic, metabolic and detoxification functions of the liver decreased. Almost complete restoration of functional activity and normalization of parameters was observed by Day 14 in EG1 animals, which was associated with the restoration of $96.15 \pm 4.31 \%$ of the initial liver weight. A prolonged decrease in protein-synthetic function is explained by pronounced reparative processes and high activity of mitotic divisions of hepatocytes, and an increase in the liver size. The pronounced proliferative activity of liver cells requires significant reserves of energy and building material; due to this fact, restoration and accumulation of protein in the body is slow.

Table 1.

Dynamics of indicators of the syndrome of hepatic cell failure (a deviation from the indicators of intact animals, \%)

\begin{tabular}{|c|c|c|c|c|c|}
\hline Time & Indices & CG1 & CG2 & EG1 & EG2 \\
\hline \multirow{5}{*}{ Day 5 } & $\mathrm{Cr}$ & $11.04 \pm 1.14$ & $14.05 \pm 1.48 *$ & $7.49 \pm 0.79 *$ & $10.21 \pm 1.17$ \\
\cline { 2 - 7 } & $\mathrm{TP}$ & $44.27 \pm 5.12$ & $38.04 \pm 4.71$ & $31.28 \pm 4.18 *$ & $31.35 \pm 3.91 *$ \\
\cline { 2 - 6 } & $\mathrm{BIN}$ & $14.11 \pm 1.05$ & $16.18 \pm 1.50$ & $10.16 \pm 1.05 *$ & $14.11 \pm 1.48$ \\
\cline { 2 - 6 } & $\mathrm{GL}$ & $21.01 \pm 3.02$ & $26.11 \pm 2.47$ & $20.48 \pm 1.74$ & $26.45 \pm 3.11$ \\
\hline \multirow{5}{*}{ Day 7 } & $\mathrm{Cr}$ & $8.47 \pm 0.58$ & $8.40 \pm 1.42$ & $\mathrm{~N}$ & $5.46 \pm 0.72 *$ \\
\cline { 2 - 6 } & $\mathrm{TP}$ & $31.16 \pm 2.14$ & $26.01 \pm 2.42 *$ & $18.98 \pm 4.16 *$ & $23.48 \pm 2.18 *$ \\
\cline { 2 - 6 } & $\mathrm{BIN}$ & $10.12 \pm 1.41$ & $9.48 \pm 1.15$ & $\mathrm{~N}$ & $9.79 \pm 1.58$ \\
\cline { 2 - 6 } & $\mathrm{GL}$ & $20.01 \pm 3.14$ & $16.12 \pm 2.00$ & $\mathrm{~N}$ & $14.56 \pm 2.08 *$ \\
\hline \multirow{5}{*}{ Day 14 } & $\mathrm{Cr}$ & $\mathrm{N}$ & $\mathrm{N}$ & $\mathrm{N}$ & $\mathrm{N}$ \\
\cline { 2 - 6 } & $\mathrm{TP}$ & $20.28 \pm 2.10$ & $18.47 \pm 1.14$ & $7.05 \pm 1.18 *$ & $12.04 \pm 1.49 *$ \\
\cline { 2 - 6 } & $\mathrm{BIN}$ & $\mathrm{N}$ & $\mathrm{N}$ & $\mathrm{N}$ & $\mathrm{N}$ \\
\cline { 2 - 6 } & $\mathrm{GL}$ & $14.11 \pm 1.57$ & $10.26 \pm 2.61$ & $\mathrm{~N}$ & $8.06 \pm 0.084 *$ \\
\hline
\end{tabular}

Note: $*_{-} P<0.05$ in comparison with $C G 1 ; N-$ Normal (indicators of intact animals)

An assessment of oxidative stress parameters showed an increase in the antioxidant content on Day 5 of the study in animals of all groups. Thus, the most pronounced activation of the protective enzymatic system was observed in EG1, which was also supported by the results of a biochemical blood test on Day 5 of the experiment (the significantly decreased cytolytic syndrome parameters and damage to hepatocytes in comparison with the control groups); on Day 7, the mentioned parameters increased. Based on the results obtained, it can be noted that due to the activation of the enzymatic antioxidant system, the content of MDA decreased, the lowest value of which was obtained in animals of EG1. The results demonstrated that cytolytic syndrome parameters were normalized in animals of EG1 by Day 7 after hepatic resection; this fact evidences the complete compensation of oxidative stress after surgery and restoration of the cell membrane integrity. On Day 14 of the study, the levels of SOD, GPx, and MDA were similar to the parameters of intact animals in both experimental and control groups (Table 2).

Studying the cytokine content, we found a significant increase in TGF- $\beta$ in animals of EG1 on Day 1 after surgery.
On Day 7, this parameter decreased insignificantly. On Day 14 after surgery, the TGF- $\beta$ level constituted $2340.74 \pm 81.12$ $\mathrm{pg} / \mathrm{ml}$ in CG1, $2107.45 \pm 107.48 \mathrm{pg} / \mathrm{ml}$ in CG2, 2537.46 \pm 78.40 $\mathrm{pg} / \mathrm{ml}$ in EG1, and $2441.12 \pm 92.12 \mathrm{pg} / \mathrm{ml}$ in EG2 (Fig.3). Thus, the maximum TGF- $\beta$ value was registered in animals of EG1. The result obtained indicates that the intrahepatic administration of cyanocobalamin activated the proliferative activity of hepatocytes. The suggested method contributes to the increased TGF- $\beta$ expression, and, at the same time, does not interrupt physiological norms of its maximum activity. A significant TGF- $\beta$ increase contributes to the most rapid restoration of the initial liver weight in animals of EG1 (up to $96.15 \pm 4.31 \%$ by Day 14 of the experiment), and results in restoration of the normal functional activity of the liver.

Table 2.

Dynamics of oxidative stress parameters

\begin{tabular}{|c|c|c|c|c|c|}
\hline Time & Indices & CG1 & CG2 & EG1 & EG2 \\
\hline \multirow{5}{*}{ Day 5} & $\begin{array}{c}\mathrm{SOD}, \\
\mathrm{pg} / \mathrm{ml}\end{array}$ & $10.80 \pm 0.24$ & $9.02 \pm 0.15$ & $13.20 \pm 1.18^{*}$ & $9.05 \pm 1.10$ \\
\cline { 2 - 6 } & $\begin{array}{c}\mathrm{GPx}, \\
\mathrm{pg} / \mathrm{ml}\end{array}$ & $0.21 \pm 0.07$ & $0.27 \pm 0.11$ & $0.36 \pm 0.18^{*}$ & $0.21 \pm 0.07$ \\
\cline { 2 - 6 } & $\begin{array}{c}\mathrm{MDA} \\
\mathrm{mmol} / 1\end{array}$ & $16.36 \pm 1.18$ & $18.97 \pm 1.24$ & $11.71 \pm 0.89 *$ & $14.27 \pm 1.05$ \\
\hline \multirow{5}{*}{ Day 7 } & $\begin{array}{c}\mathrm{SOD}, \\
\mathrm{pg} / \mathrm{ml}\end{array}$ & $12.25 \pm 1.08$ & $14.00 \pm 1.11$ & $17.14 \pm 1.24 *$ & $13.11 \pm 1.01 *$ \\
\cline { 2 - 6 } & $\begin{array}{c}\mathrm{GPx}, \\
\mathrm{pg} / \mathrm{ml}\end{array}$ & $0.28 \pm 0.05$ & $0.28 \pm 0.08$ & $0.39 \pm 0.14 *$ & $0.21 \pm 0.09 *$ \\
\cline { 2 - 6 } & $\begin{array}{c}\mathrm{MDA}, \\
\mathrm{mmol} / 1\end{array}$ & $14.21 \pm 1.07$ & $16.21 \pm 1.36$ & $7.84 \pm 0.58^{*}$ & $11.46 \pm 1.12 *$ \\
\hline \multirow{5}{*}{ Day 14 } & $\mathrm{SOD}$ & $\mathrm{N}$ & $\mathrm{N}$ & $\mathrm{N}$ & $\mathrm{N}$ \\
\cline { 2 - 6 } & $\mathrm{GPx}$ & $\mathrm{N}$ & $\mathrm{N}$ & $\mathrm{N}$ & $\mathrm{N}$ \\
\cline { 2 - 6 } & $\mathrm{MDA}$ & $\mathrm{N}$ & $\mathrm{N}$ & $\mathrm{N}$ & $\mathrm{N}$ \\
\hline
\end{tabular}

Note: ${ }^{*} P<0.05$ in comparison with $C G 1 ; N-$ Normal (indicators of intact animals)

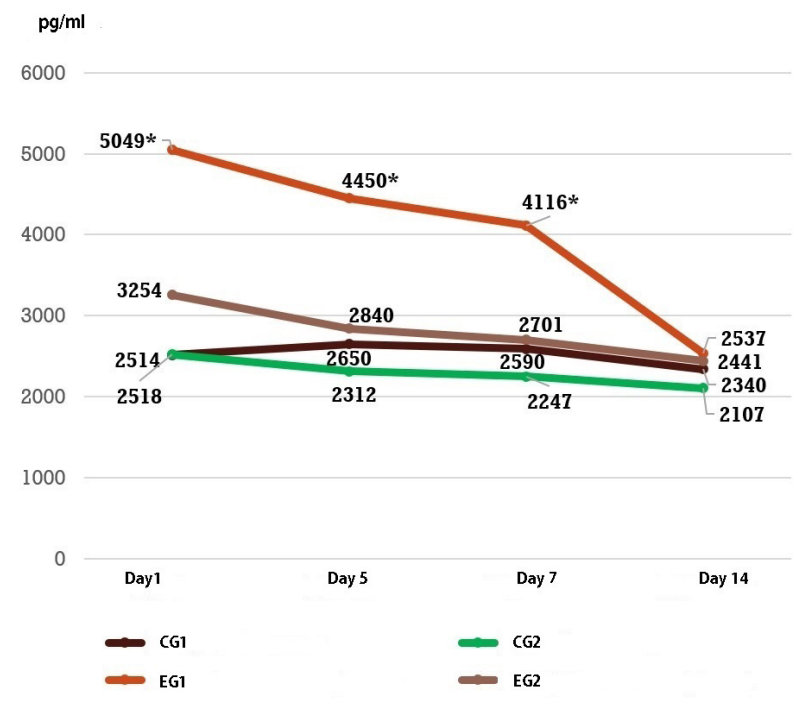

Fig. 3. Dynamics of the TGF- $\beta$ expressioncytolysis syndrome, \% 
Thus, the method suggested for prevention of the post-resection ALF using intraoperative administration of cyanocobalamin reduces the time necessary for postoperative, reparative liver regeneration. The intrahepatic method of drug administration appears to be the most optimal, since it has demonstrated the highest pharmacological efficacy compared with intraperitoneal drug administration. Intraoperative, intrahepatic administration of cyanocobalamin can increase the TGF- $\beta$ expression by 2.5-3 times, affecting the proliferative activity of hepatocytes and providing recovery to $96.15 \pm 4.31 \%$ of the initial liver weight by Day 14 after hepatectomy. Intrahepatic administration of cyanocobalamin results in activation of the antioxidant enzymatic defense system; this allows avoiding lipid peroxidation of cell membranes and reducing the severity of the cytolytic syndrome. The suggested method of administration does not cause cholestasis and contributes to the almost complete restoration of the synthetic, metabolic and detoxification functions of the liver by Day 7 after the operation.

Thus, intraoperative, intrahepatic administration of cyanocobalamin prevents the development of ALF in the early postoperative period and ensures restoration of the anatomical and functional integrity of the liver.

\section{Competing Interests}

The authors declare that they have no competing interests.

*Corresponding author: Anton P. Ostroushko, PhD. Department of general surgery, N.N. Burdenko Voronezh State Medical University. Voronezh, the Russian Federation. E-mail: antonostroushko@yandex.ru

\section{References}

1. Andreev AA, Ostroushko AP, Laptiyova AY, Gluhov AA. [Reparative hepatic regeneration after segmental resection (review article)]. Aspirantskij Vestnik Povolzh ya. 2018;5-6: 183-190. [Article in Russian].

2. Rutkin IO, Popov SA, Moiseenko VE, Biketov MA, Timergalin IV, Granov DA. [Endovideosurgical resections of the liver: experience of "Russian scientific center of radiology and surgical technologies named after acad. A. M. Granov."] Vestn Khir Im I I Grek. 2018;177(5):47-52. [Article in Russian]. 3. Ermolaev PA, Khramyh TP, Barskaya LO. [Mechanisms of damage and compensation of the systemic hemodynamics after maximally permissible hepatic resection in rats]. Omskij Nauchnij Vestnik. 2015;144(2):85-88. [Article in Russian].

4. Ahmedov SM, Safarov BD, Rasulov NA, Tabarov ZV. [Extensive hepatic resection in complicated locally advanced liver cancer]. Annals of HPB Surgery. 2014;19(4):26-31. [Article in Russian].

5. Ahmetzyanov FSh, Idrisov MN. [Methods of hepatic resection]. Kazanskij Medicinskij Zhurnal. 2015;96(4):563567. [Article in Russian].

6. Kotenko OG, Kondratyuk VA, Fedorov DA, Grinenko AV, Korshak AA, Gusev AV, Popov AO, Grigoryan MS. [Embolization of the portal vein branches in preparing patients for extensive liver resection]. Annals of HPB Surgery. 2014; 19(4):21-25. [Article in Russian].

7. Bhat M, Pasini E, Baciu C, Angeli M, Humar A, Macparland S, et al. The basis of liver regeneration: A systems biology approach. Ann Hepatol. 2019;18(3):422-428. doi:10.1016/j.aohep.2018.07.003

8. Khoronenko VE, Donskova YS, Baskakov DS, Drozhzhina OV, Sidorov DV, Lozhkin MV, et al. [Prophylaxis of hepatic insufficiency after major hepatic resections]. Anesteziologiya i Reanimatologiya. 2014;4:33-38. [Article in Russian].

9. Higgins GM. Experimental pathology of the liver. I. Restoration of the liver of the white rat following partial surgical removal. Arch Pathol. 1931;12:186-202. 